\title{
Mecanismo Eficiente de Localização Cooperativa para Veículos Autônomos Conectados
}

\author{
Wellington Lobato Junior ${ }^{1}$, Allan M. de Souza ${ }^{1}$, Eduardo Cerqueira ${ }^{2}$, \\ Denis Rosário $^{2}$, Leandro Villas ${ }^{1}$ \\ ${ }^{1}$ Instituto de Computação - Universidade Estadual de Campinas (UNICAMP) \\ ${ }^{2}$ Universidade Federal do Pará (UFPA) \\ $\{$ wellington, allan\}@lrc.ic.unicamp.br, \\ \{cerqueira, denis\}@ufpa.br, leandro@ic.unicamp.br
}

\begin{abstract}
The robust and precise location is fundamental to guarantee the functioning of Connected Autonomous Vehicles (CAVs) applications. However, vehicular navigation mechanisms (GNSS) are affected by several urban areas, mainly due to the refraction or reflection of satellite signals in construction. Thus, to use the GNSS location and satisfy the requirements of the CAV applications, it is necessary to consider data fusion techniques to obtain a more efficient positioning through the exchange of data between the CAVs. In this scenario, this article presents a data fusion mechanism for cooperative vehicular localization, called DUELAR. The mechanism considers the Kalman No Smell Filter combined with a track level map matching algorithm to correct the location errors. The simulation results showed that the DUELAR reduces the location error by at least $70 \%$ compared to other approaches in the literature.
\end{abstract}

Resumo. A localização robusta e precisa é fundamental para garantir o funcionamento de aplicações de Veículos Autônomos Conectados (CAVs). No entanto, os mecanismos de navegação veicular (GNSS) são afetados por diversos problemas nas áreas urbanas, principalmente devido à refração ou reflexão dos sinais de satélite em prédios. Assim, para usar a localização do GNSS e satisfazer os requisitos das aplicações de CAVs, é necessário considerar técnicas de fusão de dados para obter um posicionamento mais eficiente por meio da troca de dados entre os CAVs. Neste cenário, este artigo apresenta um mecanismo de fusão de dados para localização veicular cooperativa, denominado de DUELAR. O mecanismo considera o Filtro de Kalman Sem Cheiro em conjunto com um algoritmo de map matching a nível de faixa para corrigir os erros de localização. Os resultados da simulação mostraram que o DUELAR reduz o erro de localização em pelo menos $70 \%$ comparado com outras abordagens da literatura.

\section{Introdução}

Nos últimos anos, tanto a academia quanto a indústria demonstraram grande interesse na pesquisa e no desenvolvimento de veículos autônomos conectados (Connected Autonomous Vehicle - CAV). A direção autônoma possibilita uma série de benefícios para a segurança e eficiência da condução e do tráfego respectivamente, assim como utiliza melhor os recursos públicos (estradas, rodovias, transporte público) [SAE 2018]. A direção 
autônoma ainda irá liberar o condutor da estressante tarefa de dirigir o veículo, o que proporcionará um alívio físico e mental [Wang et al. 2018].

Nesse contexto, a localização robusta e precisa é fundamental e crucial para aplicações de CAVs. Por exemplo, as aplicações de sistemas avançados de assistência ao motorista (Advanced Driver Assistance System - ADAS), sistema de prevenção de colisão, blind crossing, predição de localização e estacionamento automático exigem precisão de pelo menos 30 centímetros [Kuutti et al. 2018]. Nesse contexto, os dados provenientes do sistema global de navegação por satélite (Global Navigation Satellite System - GNSS) estão inserido em todos os blocos básicos de sistemas dos CAVs para atingir os mais altos níveis de autonomia dos CAVs [SAE 2018].

No entanto, o GNSS tem algumas limitações e desafios, tais como, bloqueio de sinal, erros de órbita de satélite e erros de relógio, atrasos troposféricos e ionosféricos, para fornecer um posicionamento altamente preciso [Sharma and Kaushik 2019]. Além disso, o GNSS também é afetado por vários problemas em áreas urbanas densas, principalmente devido à refração ou reflexão de sinais de satélite em prédios [Ansari 2019]. Tais problemas causam um erro de localização variando de 5 a 30 metros, o que pode ocasionar decisões de direção erradas nos CAVs devido o GNSS fornecer a localização incorreta [Kuutti et al. 2018]. Portanto, é necessário mitigar tais desafios de sistemas de localização a fim de satisfazer os requisitos das aplicações de CAVs.

Vários trabalhos foram propostos para resolver o problema de localização, onde algumas propostas utilizam a comunicação entre veículos para permitir a troca de informações e aumentar a confiabilidade dos dados [Lobo et al. 2019a]. Por exemplo, cada veículo compartilha suas informações de localização, velocidade, aceleração e direção com os vizinhos para oferecer um posicionamento cooperativo [de Ponte Müller et al. 2016]. Além dessas informações, outros tipos de sensores de bordo podem ser utilizados, tais como, câmera, RADAR, LiDAR e Unidades de Medição Inercial (Inertial Measurement Unit - IMU) [Ansari 2019]. Desta forma, é possível combinar as informações do CAV e dos seus vizinhos por meio de técnicas de fusão de dados para obter um posicionamento mais eficiente [Hossain et al. 2019]. Os mecanismos de fusão de dados são de suma importância para integrar dados de diferentes fontes, e assim prover maior confiabilidade, exatidão e a precisão das informações de localização fornecidas para as aplicações de CAVs. Na fusão de dados, é possível combinar dados de várias fontes de forma que a incerteza de estimativas resultantes seja menor do que a incerteza de cada uma das fontes de dados individualmente [de Ponte Müller 2017]. Portanto, é importante projetar um mecanismo eficiente de fusão de dados, considerando dados provenientes de várias fontes para prover informação de localização para CAVs.

Além da fusão de dados, outras técnicas também devem ser consideradas para resolver problemas de imprecisão dos sistemas de localização, tais como map matching e mapas de alta definição [Chao et al. 2020]. O map matching é um tipo de solução que considera o processo de associação da posição a uma estrada em um mapas de alta definição [Li et al. 2017]. Uma série de etapas de pré-processamento são necessárias para corrigir os erros de trajetória, devido à imprecisão intrínseca dos sistemas de posicionamento descritos anteriormente. $\mathrm{O}$ algoritmo de map matching alinha seus dados de posicionamento à via subjacente, por exemplo, uma abordagem mais simples ajusta a posição estimada atual do CAVs com base nos pontos mais próximos em uma representação 
vetorial de uma estrada no mapa [Chao et al. 2020].

A maioria dos mecanismos propostos focaram em abordagens de fusão de dados devido às limitações de um determinado tipo de sensor que são superadas com os pontos fortes de outro sensor complementar [Wan et al. 2018] [Lobo et al. 2019a] [Nascimento et al. 2018] [Li et al. 2018]. Contudo, o benefício dos algoritmos de map matching não foi considerado e avaliado de forma adequada. Além disso, é importante considerar o trade-off entre o custo computacional das técnicas de fusão de dados e map matching e o tempo de resposta do sistema. No entanto, mecanismos de fusão de dados combinados a algoritmos de map matching para garantir os requisitos de localização para aplicações de CAVs não é uma tarefa trivial e ainda é um problema em aberto.

Para mitigar a tais problemas de localização para CAVs, neste artigo é apresentado um mecanismo de fusão de dados para localização veicular cooperativa, denominado de DUELAR. O mecanismo requer a troca de dados entre os CAVs, tais como: localização, direção, distância entre CAVs adjacentes e, também, dados fornecidos por sensores de bordo presentes no próprio CAV. O DUELAR aplica um algoritmo de fusão de dados de diversas fontes. O DUELAR também considera um algoritmo de map matching na imagem da câmera frontal de um CAV, onde os limites que constituem a pista são usados para reduzir os erros da localização estimada pelo algoritmo de fusão dos dados. Dessa forma, o DUELAR atende aos requisitos de erro de localização das aplicações de CAVs, i.e., erros de localização menor que 30 centímetros. Os resultados mostram que o DUELAR é capaz de reduzir as imprecisões de localização em até $85 \%$ e 72,52\%, em termos de erro médio absoluto (Mean Absolute Error - MAE) e raiz do erro quadrático médio (Root-Mean-Square Error - RMSE), em comparação com outros trabalhos da literatura, i.e., VLOCI, CoVaLID e Filtro de Partículas.

O restante deste artigo está organizado conforme descrito a seguir. A Seção 2 apresenta os principais trabalhos relacionados a esta pesquisa. A Seção 3 introduz o modelo de sistema e detalhes do mecanismo DUELAR. A Seção 4 apresenta a metodologia utilizada para avaliação do mecanismo, bem como a discussão acerca dos resultados obtidos. Por fim, a Seção 5 conclui o artigo.

\section{Trabalhos Relacionados}

Lobo et al. propuseram uma abordagem baseada em fusão de dados para reduzir erros de localização chamada de CoVaLID [Lobo et al. 2019a]. O trabalho considera que veículos podem transmitir dados de localização e distância dos veículos próximos, os quais servem de entrada para um algoritmo de filtro Kalman estendido (Extended Kalman filter EKF). Um modelo de média ponderada nas posições dos veículos é usado para aumentar a confiança nos dados de distância fornecidos por veículos mais próximos e aproveitar esses sensores extras para propor uma fusão de dados baseada na distância considerando o algoritmo EKF. Os resultados apresentados pelo CoVaLID tiveram um erro de até 1 metro em comparação com a localização real dos veículos, os autores aplicaram map matching para restringir os pontos estimados pelo mecanismo de fusão de dados.

Golestan et al. demonstraram dois métodos de fusão de dados usando dados do Sistema de Posicionamento Global (Global Positioning System - GPS) em conjunto com diferentes tipos de sensores presentes no veículo, tais como sensores de roda, gás e pedal de freio [Golestan et al. 2012]. Este trabalho aplicou o EKF e um filtro de partículas (Par- 
ticle filter - PF) para integrar os dados disponíveis e melhorar cooperativamente a precisão dos dados de localização dos veículos. Seus resultados mostram que o compartilhamento dos dados de localização pode garantir dados fornecidos pelo GNSS mais precisos, bem como robusto a falhas ou imprecisões do sensor.

Farhan et al. apresentaram o algoritmo VLOCI, onde veículos trocam dados de posição fornecidos pelo GNSS [Ahammed et al. 2010]. O VLOCI assume que todos os veículos podem medir as distâncias entre si, além disso, os veículos estão viajando em uma faixa e seguindo na mesma direção. O VLOCI assume que veículos mais distantes têm uma probabilidade maior de coletar dados mais errôneos, e assim o VLOCI aplicou uma técnica de média ponderada para usar os dados dos veículos mais próximos. Os resultados mostram a acurácia de 2.7 metros do VLOCI em relação aos dados de GNSS.

Liborio et al. introduziu um mecanismo de posicionamento para resolver problemas de falha crítica para o GNSS, como aqueles encontrados em ambientes com falta de sinal do GNSS, como áreas urbanas densas e túneis urbanos [Nascimento et al. 2018]. Estes cenários são caracterizados e avaliados pelos autores, que então aplicam um mecanismo de Dead Reckoning e comunicação entre veículos para resolver esses problemas. O algoritmo calibra periodicamente os sensores e usa a Geometria de Diluição da Precisão (Geometric Dilution of Precision - GDOP) para selecionar os nós com a melhor capacidade de localização. Os resultados mostram erros de aproximadamente 3 metros.

Li et al. propôs uma formalização do problema de integridade de map matching [Li et al. 2018]. Além de um método sequencial usando um PF, fornecendo um conjunto confiável de hipóteses de map matching. O algoritmo descrito pelo autor é diferente do uso mais comum de map matching para aplicações veiculares e fornece estimativas de múltiplas posições. Os autores avaliam o método usando dados adquiridos em vias públicas fornecidas pela Pacpus ${ }^{1}$.

Kang et al. desenvolveu um método de localização baseado em mapa usando uma única câmera [Kang et al. 2020]. O método usa a transformada de Hough para detectar as linhas da faixa, além de adquirir informações de direção em relação à faixa em que o veículo está se movendo. $\mathrm{O}$ autor também emprega o algoritmo de map matching baseado no ponto mais próximo iterativo para determinar e eliminar a disparidade entre as trajetórias de GNSS e o mapa de referência.

Hansson et al. propôs um modelo oculto de Markov para combinar uma trajetória de medições GNSS ruidosas com as pistas nas quais o veículo registra suas posições [Hansson et al. 2020]. O modelo depende de um sinal de mudança de faixa, bem como de informações visuais sobre marcações de faixa próximas. $\mathrm{O}$ autor avalia o modelo em um mapa HD fornecido pela TomTom e os dados do sensor do veículo são extraídos do projeto Drive Me da Volvo [Cars 2015].

Com base na análise do estado da arte, identificou-se que a maioria dos trabalhos de map matching não considera a possibilidade de usar localização dos CAVs vizinhos para aumentar a precisão da posição [Li et al. 2018] [Kang et al. 2020] [Hansson et al. 2020]. Além disso, os trabalhos de localização estão restritos ao comportamento de fusão de dados em um cenário de comunicação veicular [Lobo et al. 2019a] [Golestan et al. 2012] [Ahammed et al. 2010] [Nascimento et al. 2018]. Os benefícios

\footnotetext{
${ }^{1}$ pacpus.hds.utc.fr
} 
dos algoritmos de map matching não foram considerados e avaliados de forma adequada. Os trabalhos de Lobo et al. e Liborio et al. não deixam claro como é feita a aplicação prática dos algoritmos. Por fim, os trabalhos que utilizam o PF não podem garantir que a convergência do filtro e o tempo de resposta atendam às aplicações CAVs, devido ao trade-off entre a precisão e o custo computacional [Balico et al. 2018]. Se o filtro aumentar o número de partículas, a precisão do preditor também aumentará. No entanto, o custo computacional e o tempo de resposta do sistema de localização também aumentam.

\section{Mecanismo de Fusão de Dados para Localização Cooperativa}

Esta Seção descreve o mecanismo DUELAR, o qual realiza a fusão de dados por meio do filtro de Kalman Sem Cheiro (Unscented Kalman Filter - UKF), considerando dados de sensores locais e dados de sensores que os CAVs transmitem periodicamente. Além disso, o mecanismo DUELAR considera um algoritmo de map matching para atualizar a localização do CAV.

\subsection{Modelo de Rede e Sistema}

Foi considerado um cenário composto de $n$ CAVs que se deslocam em uma área urbana ou rodoviária com várias pistas, onde cada veículo tem uma identidade individual $(i \in$ $[1, n]$ ) e é equipado com um rádio que possibilita a comunicação entre veículos (V2V, de Vehicle-to-Vehicle). Esses veículos são representados em um grafo dinâmico $G(V, E)$, onde os vértices $V=\left\{v_{1}, \cdots, v_{n}\right\}$ representam um conjunto finito de veículos, e as arestas $E=\left\{e_{1}, \cdots, e_{m}\right\}$ constrói um conjunto finito de enlaces sem fio assimétricos entre veículos vizinhos.

Cada CAV $v_{i}$ é equipado com uma câmera frontal, capaz de fornecer imagens do campo de Visão (Field of View - FoV) para as diferentes aplicações dos CAVs. A Figura 1(a) apresenta o funcionamento da câmera frontal para detectar CAV (bounding box) por meio de algoritmos de processamento de imagem para ser usado pelas aplicações dos CAVs. Também é possível observar na Figura 1(b), a aplicação da detecção da faixa para ser utilizada com o algoritmo de map matching e outros sistemas de direção autônoma.

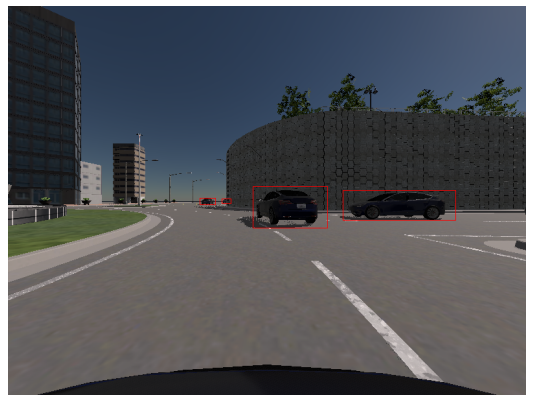

(a) Detecção do Bounding Box dos CAVs

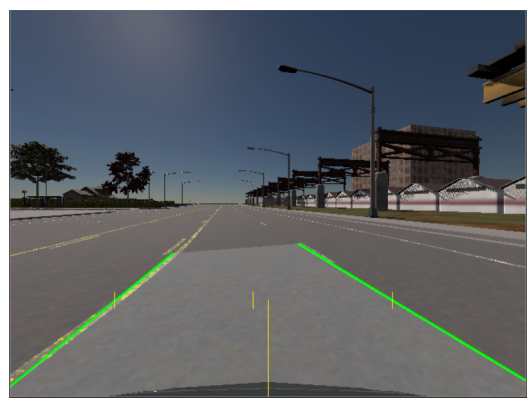

(b) Detecção da Faixa

Figura 1. Detecção de CAVs e Faixa pela Câmera RGB Frontal

Cada veículo $v_{i}$ se move em direção a uma certa direção $\left(\overrightarrow{\operatorname{dir}_{i}}\right)$ seguindo um caminho de viagem predefinido $R_{i}$ com velocidade $s_{i}$ variando entre um mínimo $\left(s_{\text {min }}\right)$, e um limite de velocidade máximo $\left(s_{\max }\right)$. Cada veículo $v_{i}$ tem uma posição $L_{i}=\left(X_{i}, Y_{i}\right)$ em 
um determinado instante de tempo $t$ atribuída pelo GNSS. Entretanto, o GNSS apresenta erros de localização intrínsecos aos sistemas de posicionamento. Foi considerado a adição de um ruído branco gaussiano de média zero $\mathcal{N}\left(\lambda, \sigma^{2}\right)$ para representar a degradação do sinal do GNSS que pode ser afetado por alguns fatores externos, tal como apresentado em [Hossain et al. 2019]. Para variar o impacto do ruído branco gaussiano de média zero, as alterações devem ser realizadas no desvio padrão, tal como apresentado nas Equações $1 \mathrm{e}$ 2. Portanto, a localização $L_{i}$ representa as coordenadas $X_{G N S S}$ e $Y_{G N S S}$.

$$
\begin{gathered}
X_{G N S S}=X+\mathcal{N}\left(\lambda, \sigma^{2}\right) \\
Y_{G N S S}=Y+\mathcal{N}\left(\lambda, \sigma^{2}\right)
\end{gathered}
$$

Os CAVs consideram o mecanismo DUELAR para mitigar os erros de localiza-

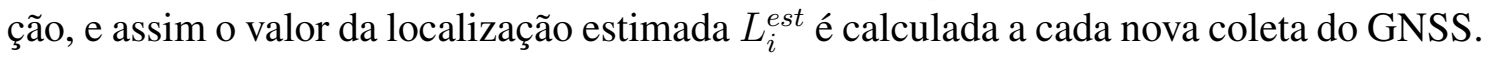
Especificamente, o mecanismo DUELAR tem o funcionamento dividido em duas etapas, onde a primeira etapa é responsável por coletar os dados dos CAVs vizinhos e utilizar a fusão de dados para reduzir os erros presentes na localização. A segunda etapa é responsável por aplicar um algoritmo de map matching para corrigir a localização estimada pela etapa anterior.

É importante ressaltar que os CAVs presentes no cenário transmitem beacons periodicamente, o que ocorre naturalmente nas aplicações veiculares. Dessa forma, o DUELAR aproveita a troca de beacons realizada entre os CAVs para disseminar os dados necessárias para o funcionamento do mecanismo, tais como, localização $L_{i}$ e direção $\overrightarrow{d i r}$. Desta forma, não é gerado sobrecarga de sinalização na rede. Ao receber um beacon de um determinado vizinho $v_{j}$, o CAV $v_{i}$ armazena, em uma tabela, os dados de localização $L_{j}$, direção $\overrightarrow{d i r_{j}}$ e a distância euclidiana $d_{i, j}^{g n s s}$. Vale a pena realçar que são armazenados apenas dados de veículos locomovendo-se na mesma direção.

Também são consideradas imagens da câmera frontal do CAV $v_{i}$ para detectar CAVs presentes no FoV (ver Figura 1(a)), e assim é possível extrair a distância euclidiana $d_{i, j}^{c a m}$ entre os CAVs por meio de algoritmos de processamento de imagem. Desta forma, é possível escolher o CAV vizinho que tem a menor distância $d_{i, j}^{c a m}$, pois a imprecisão será maior para distâncias $d_{i, j}^{c a m}$ maiores [Lobo et al. 2019b]. O CAV vizinho $v_{j}$ com menor distância $d_{i, j}^{c a m}$ detectada pela câmera deve ser associado com uma localização $L_{j}$. Para que isso ocorra, a tabela que armazena os dados compartilhados pelos CAVs vizinhos é percorrida, e a tupla da tabela com valor de $d_{i, j}^{g n s s}$ mais próximo a $d_{i, j}^{\text {cam }}$ é selecionada. Como existe divergência entre os valores de distância calculada com base na localização fornecida pelo GNSS $d_{i, j}^{\text {gnss }}$ e a distância calculada com base em algoritmo de processamento de imagens $d_{i, j}^{c a m}$, é necessário a utilização de técnica de similaridade de triângulo para calcular a posição $L_{j}^{\prime}$ do CAV $v_{j}$. Por fim, os valores de localização $L_{i}$ do CAV $v_{i}$, a localização $L_{j}^{\prime}$ do CAV vizinho $v_{j}$ e a distância $d_{i, j}^{c a m}$ entre os CAVs $v_{i}$ e $v_{j}$ são utilizados como entrada para o funcionamento do algoritmo de fusão de dados.

\subsection{Fusão de dados}

Foi escolhido o UKF como algoritmo de fusão de dados para estimar a localização $L_{i}^{\text {est }}$ de um CAV $v_{i}$. O UKF pertence a classes dos filtros de Kalman e adiciona os pontos sigma 
para representar a distribuição da variável coletada, i.e., $L_{i}, L_{j}^{\prime}$ e $d_{i, j}^{c a m}$. Foi utilizado o UKF para evitar imprecisões e limitações encontrados em outros filtros, pois o UKF considera um conjunto de pontos sigma para calcular a média e a covariância das variáveis de entrada. Esses pontos sigma são escolhidos com base em uma função que leva em consideração as características do problema modelado. Desta forma, o UKF usa pontos sigma, mas reduz drasticamente a quantidade de cálculo usando um método determinístico para escolher os pontos que serão usados.

Os pontos sigma são as amostras que caracterizam a média e a covariância das variáveis de entrada $x=\left[L_{i}, L_{j}^{\prime}, d_{i, j}^{c a m}\right]$. De acordo com uma função, essas amostras ou pontos são escolhidos deterministicamente e é atribuído um peso para cada ponto. Foi utilizada a função descrita por [Wan and Van Der Merwe 2000], a qual requer os parâmetros $\alpha, \beta$ e $\kappa$ para definir os pontos sigma $\mathcal{X}$, além dos pesos $W^{m}$ e $W^{c}$. O parâmetro $\alpha$ determina a propagação dos pontos sigma em torno da matriz média de entrada $\bar{x}$, $\beta$ é usado para incorporar conhecimento prévio sobre $x$, e $\kappa$ é um parâmetro de escala secundário que geralmente é definido como 0.

$$
\mathcal{X}, W^{m}, W^{c}=\text { Merwe }^{\prime} \text { SigmaFunction }(\alpha, \beta, \kappa)
$$

O UKF representa um conjunto de equações que constitui um procedimento recursivo de estimação, a qual tende a estar mais próxima dos valores reais do que as medições iniciais. Isso deve-se ao fato que a média ponderada tem uma incerteza de estimativa melhor do que qualquer um dos valores individuais que realizam a mesma média. Observando a variável de entrada, é possível estimar estados passados, presentes e futuros para encontrar a variável de saída $L_{i}^{\text {est }}$. O UKF possui duas etapas: previsão e atualização.

Na etapa de previsão, os pontos sigma $\mathcal{X}$ são propagados no próprio sistema não linear que representa o modelo e, após esta fase, capturam com precisão a covariância média posterior, utilizou-se a notação $\Upsilon_{i}$ para representar os pontos sigma após essa fase. A fase inicial do filtro calcula $2 n+1$ pontos ponderados $W^{m} \Upsilon$, onde $n$ é a dimensão das variáveis de entrada, a matriz média de entrada $\bar{x}$ e a matriz de covariância $\bar{P}$ são determinados a partir das Equações 4 e 5 . A matriz de medida estimada $\mu_{\mathcal{Z}}$ é calculada pelos pontos sigma aplicados no modelo de medição $x$.

$$
\begin{gathered}
\bar{x}=\sum_{i=0}^{2 n} W_{i}^{m} \Upsilon_{i} \\
\bar{P}=\sum_{i=0}^{2 n} W_{i}^{c}\left(\Upsilon_{i}-\bar{x}\right)\left(\Upsilon_{i}-\bar{x}\right)^{T}+Q
\end{gathered}
$$

A média dos vetores das medidas $\mu_{\mathcal{Z}}$ e a covariância das medidas $P_{\mathcal{Z}}$, são calculadas com base nas estatísticas dos pontos transformados, conforme pode ser observado nas Equações 6 e 7. Esses valores são usados na fase de atualização.

$$
\mu_{\mathcal{Z}}=\sum_{i=0}^{2 n} W_{i}^{m} \mathcal{Z}_{i}
$$




$$
P_{\mathcal{Z}}=\sum_{i=0}^{2 n} W_{i}^{c}\left(\mathcal{Z}_{i}-\mu_{\mathcal{Z}}\right)\left(\mathcal{Z}_{i}-\mu_{\mathcal{Z}}\right)^{T}+R
$$

Após a fase de previsão ser concluída, as equações de atualização de medição são usadas para determinar a nova estimativa dos valores de entrada $x$ e a covariância $P$ de acordo com o ganho de Kalman mostrado na Equação 8. A Equação 9 calcula a nova estimativa da localização $L_{i}^{e s t}$ usando a matriz média de entrada $\bar{x}$ e o ganho de Kalman $K$, enquanto que a Equação 10 calcula a nova covariância. Desta forma, o filtro mantém as etapas de previsão e atualização até o final da medição do estado. Depois disso, os pontos são corrigidos usando o algoritmo de correspondência de mapa no nível da pista.

$$
\begin{gathered}
K=\left[\sum_{i=0}^{2 n} W_{i}^{c}\left(\Upsilon_{i}-\bar{x}\right)\left(\mathcal{Z}_{i}-\mu_{\mathcal{Z}}\right)^{T}\right] P_{\mathcal{Z}}^{-1} \\
x=\bar{x}+K\left(z-\mu_{\mathcal{Z}}\right) \\
P=\bar{P}-K P_{\mathcal{Z}} K^{T}
\end{gathered}
$$

\subsection{Lane Level Map Matching}

Para associar determinada localização $L_{i}^{\text {est }}$ ruidosa com uma determinada faixa de um mapa é necessário utilizar informações de mapas de alta definição. Uma vez que as coordenadas de cada estrada em que o CAV se encontra são conhecidas. Este algoritmo de map matching reduz a área de erro, pois é limitado a apenas posições restritas a uma determinada faixa. Para identificar a via, foi utilizado uma abordagem semelhante ao trabalho de [Kang et al. 2020], onde foi implementado com uma câmera RGB frontal.

Uma vez identificada a faixa onde o veículo está inserido, pode-se considerar essa faixa como uma linha composta por duas tuplas de pontos $A=\left(X_{A}, Y_{A}\right)$ e $B=\left(X_{B}, Y_{B}\right)$. Dada uma terceira tupla representando o ponto resultante do mecanismo de fusão de dados $L_{i}^{e s t}=X_{i}^{\text {est }}, Y_{i}^{\text {est }}$, é aplicado o Algoritmo 1 para recuperar a distância mínima entre um ponto e uma linha. Dessa forma, o resultado é uma nova localização estimada $L_{i}^{\text {est’ }}$ que está dentro das limitações da faixa e pode ser usado para a identificar a localização do CAV $v_{i}$.

\section{Avaliação}

Esta Seção apresenta a avaliação do mecanismo DUELAR em comparação com o GPS, VLOCI, CoVaLID e PF. Além disso, são apresentados a descrição do cenário de avaliação, as configurações utilizadas e os resultados obtidos em termos de RMSE e MAE.

\subsection{Descrição do Cenário de Avaliação}

Para avaliar o mecanismo desenvolvido, foi utilizado o framework Open Source Veins ${ }^{2}$ do OMNeT $++^{3}$ que implementa a pilha de protocolo do padrão IEEE $802.11 \mathrm{p}$ para comunicação entre veículos e um modelo de obstáculo para atenuação de sinal. Também

\footnotetext{
${ }^{2}$ http://veins.car2x.org/

${ }^{3}$ http: //omnetpp.org/
} 


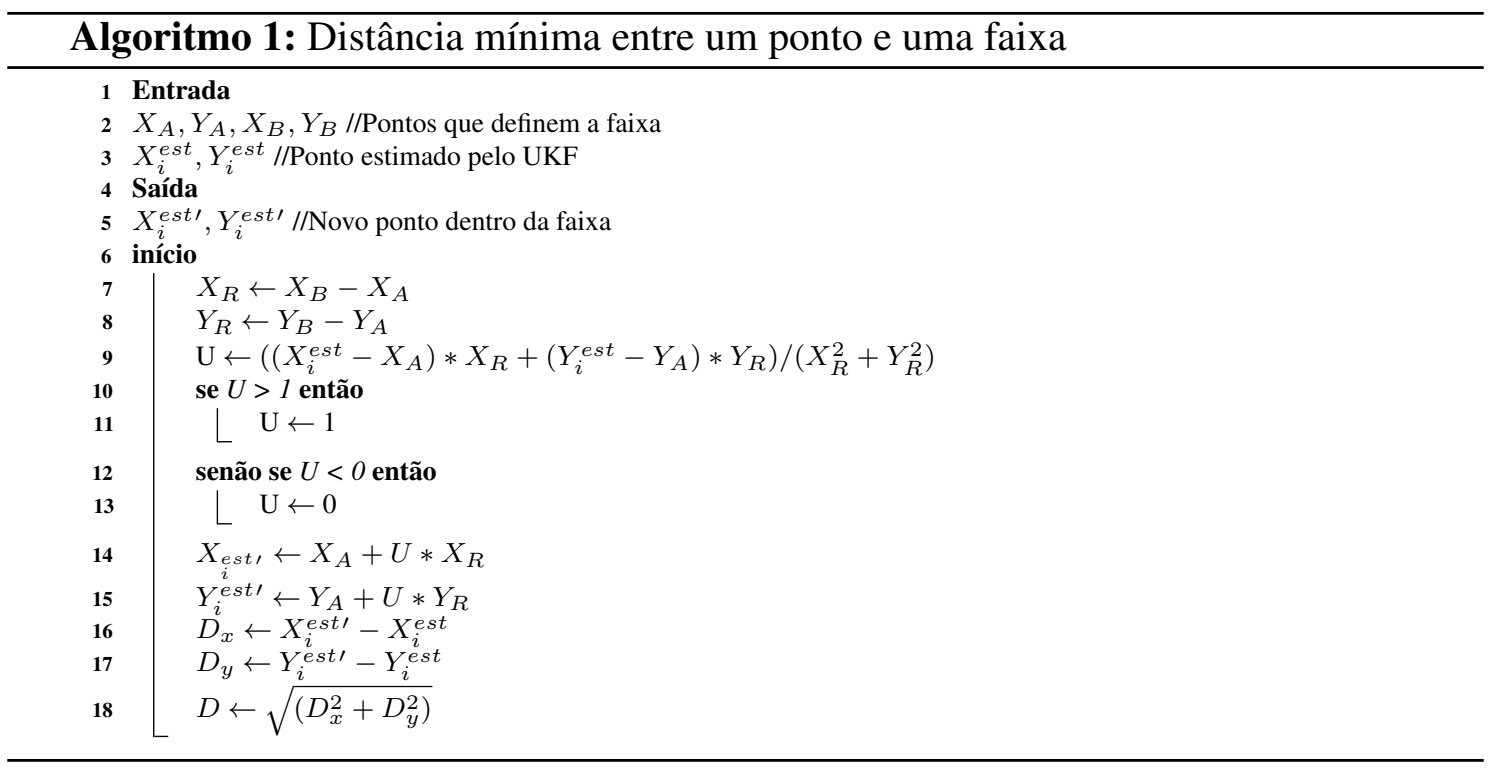

foi utilizado o simulador Simulation of Urban MObility (SUMO) ${ }^{4}$ e o CARLA ${ }^{5}$ para simular o tráfego e a mobilidade dos CAVs. Isso nos permite reproduzir os movimentos dos CAVs desejados com velocidade de cruzeiro aleatória e interações V2V de acordo com dados empíricos. Foram realizadas 50 simulações com diferentes sementes geradas aleatoriamente, e os resultados apresentaram valores com intervalo de confiança de $95 \%$.

CARLA é um simulador construído para análise de direção autônoma e totalmente open source. $\mathrm{O}$ ambiente de simulação fornece um cenário de direção urbana preenchido com edifícios, veículos, pedestres e cruzamentos [Dosovitskiy et al. 2017]. O simulador contém a definição de vários cenários que podem ser usados para avaliar o comportamento de aplicações CAV. Nas simulações, foi considerado o cenário Town 3 conforme mostrado na Figura 2. O cenário Town 3 é o mais complexo disponível no simulador CARLA e inclui um cruzamento de 5 faixas, uma rotatória, desnível, um túnel e Urban Street Canyons, possibilitando testes em diferentes situações mais realistas.

Os CAVs usados na simulação compartilham as mesmas características, como mesmo tamanho, velocidade e aceleração média. Os CAVs também possuem uma câmera fixada na frente para prover imagens RGB para detecção de pista e objetos (i.e., CAVs), além do GPS para capturar as posições e IMU para capturar a direção do veículo. Todos os principais parâmetros de simulação estão resumidos na Tabela 1.

O mecanismo DUELAR foi comparado com outros mecanismos para corrigir os erros de localização. Mais especificamente, o GPS é a posição original coletada pelo GNSS com ruído branco gaussiano de média zero, sendo esta o baseline para comparar com os outros mecanismos. O erro médio de localização para esta simulação está entre 5 a 10 metros. O CoVaLID [Lobo et al. 2019a] utiliza os dados dos veículos vizinhos aplicados no EKF em conjunto com o algoritmo de map matching. No entanto, a abordagem considera o ponto mais próximo da via e não o ponto mais próximo da faixa. $\mathrm{O}$ PF [Golestan et al. 2012] é uma abordagem utilizando o Filtro de Partículas para realizar

\footnotetext{
${ }^{4}$ http: // sumo.dlr.de

${ }^{5}$ http://carla.org/
} 
a fusão dos dados. Por fim, o VLOCI [Ahammed et al. 2010] aplica uma heurística nos dados de posição e distância de outros veículos para corrigir as localizações.

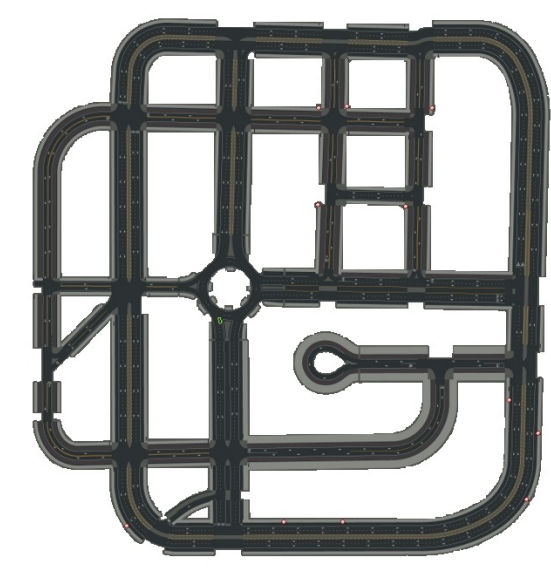

Figura 2. Topologia do cenário CARLA da Town 3

\begin{tabular}{cc}
\hline Parâmetro & Valor \\
\hline Densidade de veículos & 50 veículos \\
Frequência do canal & $5.89 \mathrm{GHz}$ \\
Potência de transmissão & $2.2 \mathrm{~mW}$ \\
Sensibilidade & $-94 \mathrm{dBm}$ \\
Raio de comunicação & $300 \mathrm{~m}$ \\
Taxa de bits & $6 \mathrm{Mbps}$ \\
Frequência de GPS & $10 \mathrm{~Hz}$ \\
Erro de GPS & $5-10 \mathrm{~m}$ \\
Tamanho do cenário & $600 \mathrm{~m} \mathrm{X} \mathrm{600} \mathrm{m}$ \\
Tempo de simulação & $200 \mathrm{~s}$ \\
$\alpha, \beta, \kappa$ & $0.001,2,0$ \\
\hline
\end{tabular}

Tabela 1. Parâmetros de Simulação para o Cenário Town 3

Com o objetivo de avaliar os erros de localização, as métricas de precisão da medida podem ser usadas para analisar, tais como RMSE e MAE. O RMSE por si só não é um bom indicador de erro de localização [Chai and Draxler 2014] e, portanto, o MAE seria uma métrica complementar para esse propósito. Tanto os resultados de RMSE quanto o MAE pertencem ao intervalo $[0, \infty]$. Eles são baseados na comparação do valor predito ou adquirido pelo mecanismo em relação ao valor real. Um menor resultado de RMSE e MAE significa melhor precisão de localização e menor erro. Outra característica crítica a ser observada nessas métricas é que os valores RMSE são iguais ou maiores do que MAE. O RMSE atribui um peso aproximadamente alto a erros significativos. Para avaliar o comportamento das abordagens, é apresentado os resultados para os eixos $x$ e $y$, além da média de ambos os eixos. Os eixos $x$ e $y$ representam as posições em termos de latitude e longitude de um CAV. Os resultados em diferentes eixos são demonstrados para apurar o impacto das abordagens de cada componente que integram a posição do CAV.

\subsection{Análise dos Resultados}

A Figura 3 apresenta os resultados do RMSE para a localização obtida por meio do GPS, VLOCI, PF, CoVaLID e DUELAR. Ao analisar os resultados da Figura 3(a), é possível notar a redução no erro de localização de $95 \%$ do DUELAR em comparação com os dados apenas do GPS e a abordagem VLOCI, além de reduzir cerca de $60 \%$ em relação ao CoVaLID e 33\% em comparação com o PF. O bom resultado do mecanismo DUELAR deve-se ao fato de usar o algoritmo UKF para realizar a fusão dos dados de diferentes fontes. Além disso, o mecanismo DUELAR considera um algoritmo de map matching a nível de faixa presente em seu mapa de alta definição, onde a identificação da faixa é realizada com o auxílio da câmera presente no CAV. Dessa forma, o DUELAR pode usar a posição estimada e associá-la ao ponto mais próximo da faixa.

O mesmo comportamento pode ser observado na Figura 3(b), onde o DUELAR reduziu o erro de localização do eixo y em cerca de $50 \%$ do erro em comparação com o CoVaLID, 75\% em comparação com o PF e 90\% em comparação com o GPS. Ainda 
é possível analisar os resultados do RMSE para ambos os eixos na Figura 3(c), onde o mecanismo DUELAR apresentou um erro de localização de 26 centímetros. Tal erro equivale a uma redução de $72,52 \%$ em relação ao CoVaLID, que é o segundo melhor mecanismo. Desta forma, é possível observar que o mecanismo DUELAR atende os requisito para a maioria das aplicações de $\mathrm{CAV}$, onde a aplicação mais desafiadora e requer erro de localização menor que 30 centímetros.

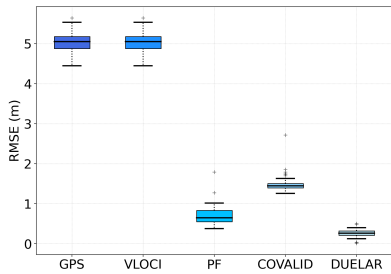

(a) Eixo $\mathrm{X}$

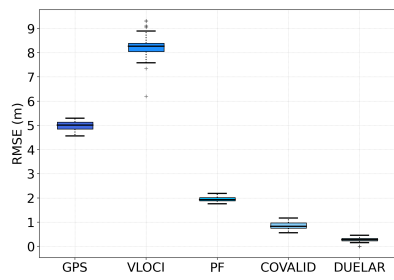

(b) Eixo Y

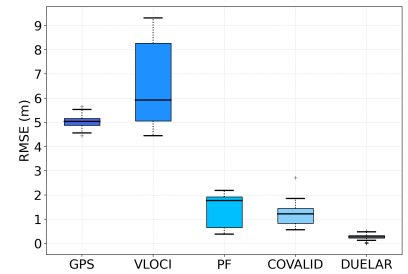

(c) Ambos os eixos

Figura 3. Resultados de RMSE no eixo $x$, eixo $y$ e em ambos os eixos para todos os veículos no cenário

Também foi avaliada a métrica de MAE para cada proposta aplicada no cenário, tal como apresentado nas Figura 4. Os valores MAE mostraram comportamento semelhante em comparação aos resultados RMSE. O mecanismo DUELAR reduz o erro de localização em até $85 \%$ em comparação com outras abordagens. É possível analisar que os quartis apresentados pelo mecanismo DUELAR em termos de MAE estão muito próximos, i.e., tem uma baixa variação. O maior valor de erro identificado na Figura 4(a) é de 43 centímetros, enquanto que o menor valor observado está em torno de 36 centímetros. Além disso, a Figura 4 mostra que para ambos os eixos, o pior resultado apresentado pelo mecanismo DUELAR é equivalente ao melhor resultado das abordagens PF e CoVaLID.

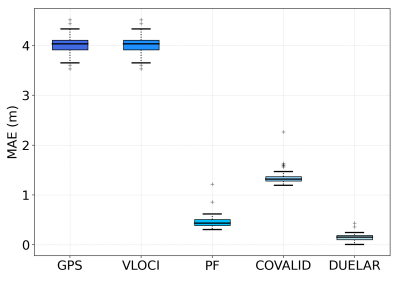

(a) Eixo $\mathrm{X}$

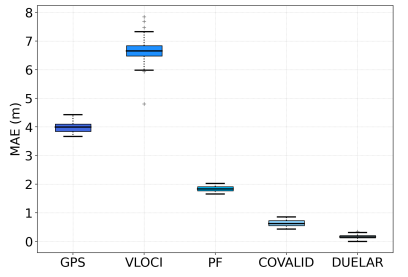

(b) Eixo Y

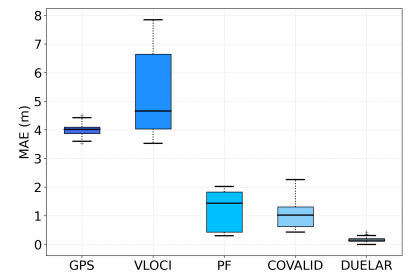

(c) MAE em ambos os eixos

Figura 4. Resultados de MAE no eixo $x$, eixo y e em ambos os eixos para todos os veículos no cenário

Os resultados de RMSE e MAE são iguais no eixo $x$ para o VLOCI e GPS, e esse comportamento já foi demonstrado em [Lobo et al. 2019a]. É necessário notar que o desempenho apresentado pelo VLOCI é inferior até mesmo que ao GPS nas Figuras 3(b), 4(b), 3(c) e 4(c). Esse desempenho ocorre devido ao fato do mecanismo VLOCI assumir que os CAVs estão trafegando em uma única faixa, na mesma direção e com a mesma velocidade, o que não ocorre necessariamente nos cenários simulados. Além disso, o VLOCI assume que o cenário é totalmente estático, sem nenhuma mobilidade veicular, 
e com um número fixo de CAVs. As considerações feitas pela abordagem VLOCI tornam o cenário muito distante do real, o que impacta negativamente no funcionamento da proposta em um cenário com mobilidade e com um número variado de CAVs.

Para demonstrar o impacto dos erros de localização na trajetória de um CAV, foi escolhida uma parte de um rota para cada abordagem avaliada como pode ser observado na Figura 5. Ao analisar a Figura 5(a), é possível observar os erros de localização do GPS com a adição do erro $\mathcal{N}$ em comparação com a localização real do CAV, i.e., ground truth. O comportamento do VLOCI é semelhante aos dados de GPS, como observado na Figura 5(b). As abordagens CoVaLID e PF apresentam resultados semelhantes e ainda é possível observar que as estimativas realizadas por ambos são alinhadas com o mapa aplicando o algoritmo de map matching. Por exemplo, o CoVaLID e PF tem um RMSE de 54 e 109 centímetros, respectivamente. Por fim, a Figura 5(e) apresenta o comportamento do mecanismo DUELAR, pode-se observar que o mecanismo obteve o melhor resultado com alguns erros pontuais apresentados na trajetória. Por exemplo, o mecanismo DUELAR teve um erro de localização de 35 centímetros para essa trajetória.

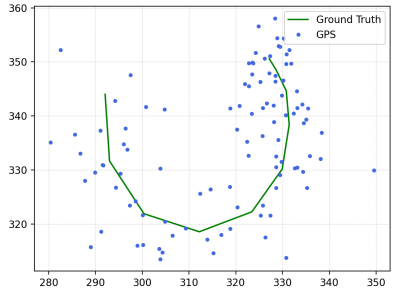

(a) GPS

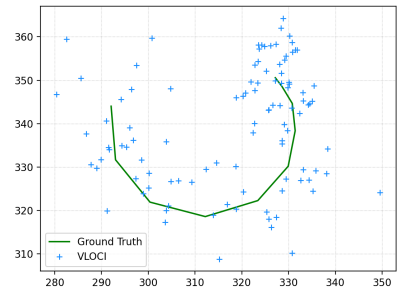

(b) VLOCI

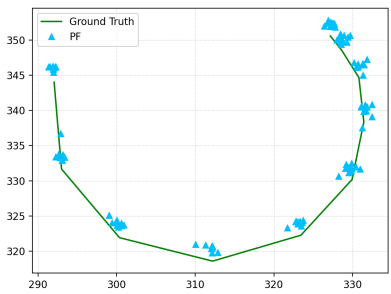

(c) PF

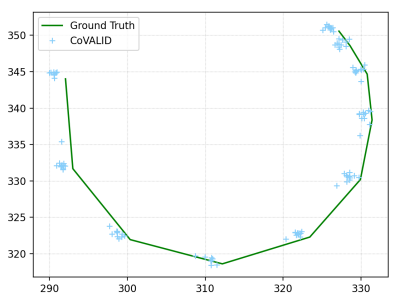

(d) CoVaLID

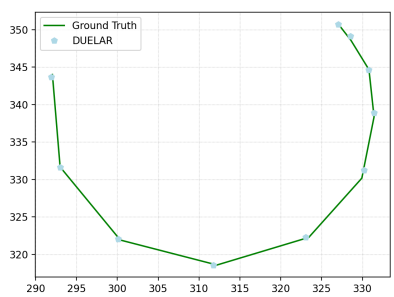

(e) DUELAR

Figura 5. Rota e a Localização de um CAV para as diferentes abordagens

Com base na análise das avaliações realizadas, foi possível concluir que o mecanismo DUELAR provê localização com baixo erros em termos de RMSE e MAE. Além disso, o DUELAR tem um desempenho superior às abordagens CoVaLID, PF e VLOCI. De acordo com os resultados, o maior erro analisado fica em torno de 50 centímetros. Esse desempenho deve-se ao fato do DUELAR considerar o UKF para realizar a fusão de dados de dados de diferentes fontes, bem como de aplicar o algoritmo de map matching no nível da pista ainda mais para reduzir o impacto dos erros na localização. Os resultados indicam que a fusão de dados e o algoritmo de map matching são técnicas complementares para resolver alguns dos problemas de localização. 


\section{Conclusão}

Este artigo apresentou o mecanismo DUELAR para reduzir o impacto dos erros na posição dos CAVs utilizando o UKF em conjunto com um algoritmo de map matching. O mecanismo DUELAR leva em consideração a posição dos CAVs vizinhos, direção e a distância estimada pela câmera para realizar a fusão dos dados. Para aumentar a precisão da localização estimada, o mecanismo ainda aplica um algoritmo de map matching levando em consideração as informações de mapas de alta definição, dessa forma, o mecanismo DUELAR associa as localizações estimadas dentro da faixa onde o CAV está posicionado. No geral, os resultados demonstram que DUELAR, garante uma posição precisa com MAE e RMSE menor em pelo menos $85 \%$ e 72,52\%, respectivamente, comparado ao comparado aos dados brutos do GPS, VLOCI, CoVaLID e PF. Devido ao mecanismo de fusão de dados e algoritmo de map matching, DUELAR aparece como uma alternativa interessante para ser executado em conjunto com outras aplicações CAVs.

\section{Agradecimento}

Este trabalho contou apoio financeiro concedido pela Fundação de Amparo à Pesquisa do Estado de São Paulo (FAPESP) por meio dos processo de no 2019/19105-3, 2019/24937-8 e 2018/19639-5.

\section{Referências}

Ahammed, F., Taheri, J., Zomaya, A. Y., and Ott, M. (2010). Vloci: Using distance measurements to improve the accuracy of location coordinates in gps-equipped vanets. In International Conference on Mobile and Ubiquitous Systems: Computing, Networking, and Services, pages 149-161. Springer.

Ansari, K. (2019). Cooperative position prediction: Beyond vehicle-to-vehicle relative positioning. IEEE Trans. on Intelligent Transportation Systems, 21(3):1121-1130.

Balico, L. N., Loureiro, A. A., Nakamura, E. F., Barreto, R. S., Pazzi, R. W., and Oliveira, H. A. (2018). Localization prediction in vehicular ad hoc networks. IEEE Communications Surveys \& Tutorials, 20(4):2784-2803.

Cars, V. (2015). Drive me.

Chai, T. and Draxler, R. R. (2014). Root mean square error (rmse) or mean absolute error (mae)?-arguments against avoiding rmse in the literature. Geoscientific model development, 7(3):1247-1250.

Chao, P., Xu, Y., Hua, W., and Zhou, X. (2020). A survey on map-matching algorithms. In Australasian Database Conference, pages 121-133. Springer.

de Ponte Müller, F. (2017). Survey on ranging sensors and cooperative techniques for relative positioning of vehicles. Sensors, 17(2):271.

de Ponte Müller, F., Diaz, E. M., and Rashdan, I. (2016). Cooperative positioning and radar sensor fusion for relative localization of vehicles. In Intelligent Vehicles Symposium, pages 1060-1065. IEEE.

Dosovitskiy, A., Ros, G., Codevilla, F., Lopez, A., and Koltun, V. (2017). Carla: An open urban driving simulator. In Conference on robot learning, pages 1-16. PMLR. 
Golestan, K., Seifzadeh, S., Kamel, M., Karray, F., and Sattar, F. (2012). Vehicle localization in vanets using data fusion and v2v communication. In symposium on Design and analysis of intelligent vehicular networks and applications, pages 123-130.

Hansson, A., Korsberg, E., Maghsood, R., Norden, E., and Selpi, S. (2020). Lane-level map matching based on hmm. IEEE Transactions on Intelligent Vehicles.

Hossain, M. A., Elshafiey, I., and Al-Sanie, A. (2019). Cooperative vehicle positioning with multi-sensor data fusion and vehicular communications. Wireless Networks, 25(3):1403-1413.

Kang, J. M., Yoon, T. S., Kim, E., and Park, J. B. (2020). Lane-level map-matching method for vehicle localization using gps and camera on a high-definition map. Sensors, 20(8):2166.

Kuutti, S., Fallah, S., Katsaros, K., Dianati, M., Mccullough, F., and Mouzakitis, A. (2018). A survey of the state-of-the-art localization techniques and their potentials for autonomous vehicle applications. IEEE Internet of Things Journal, 5(2):829-846.

Li, F., Bonnifait, P., and Ibañez-Guzmán, J. (2018). Map-aided dead-reckoning with lane-level maps and integrity monitoring. IEEE Transactions on Intelligent Vehicles, 3(1):81-91.

Li, F., Bonnifait, P., Ibanez-Guzman, J., and Zinoune, C. (2017). Lane-level mapmatching with integrity on high-definition maps. In Intelligent Vehicles Symposium, pages 1176-1181. IEEE.

Lobo, F., Grael, D., Oliveira, H., Villas, L., Almehmadi, A., and El-Khatib, K. (2019a). Cooperative localization improvement using distance information in vehicular ad hoc networks. Sensors, 19(23):5231.

Lobo, F. L., Grael, D. C., Oliveira, H. A. d., Villas, L. A., Almehmadi, A., and El-Khatib, K. (2019b). A distance-based data fusion technique for minimizing gps positioning error in vehicular ad hoc networks. In 15th International Symposium on QoS and Security for Wireless and Mobile Networks, pages 101-108.

Nascimento, P. P. L. L. d., Kimura, B. Y. L., Guidoni, D. L., and Villas, L. A. (2018). An integrated dead reckoning with cooperative positioning solution to assist gps nlos using vehicular communications. Sensors, 18(9):2895.

SAE, J. (2018). 3016-2018, taxonomy and definitions for terms related to driving automation systems for on-road motor vehicles. Society of Automobile Engineers, sae.

Sharma, S. and Kaushik, B. (2019). A survey on internet of vehicles: Applications, security issues \& solutions. Vehicular Communications, 20:100182.

Wan, E. A. and Van Der Merwe, R. (2000). The unscented kalman filter for nonlinear estimation. In Adaptive Systems for Signal Processing, Communications, and Control Symposium, pages 153-158. IEEE.

Wan, G., Yang, X., Cai, R., Li, H., Zhou, Y., Wang, H., and Song, S. (2018). Robust and precise vehicle localization based on multi-sensor fusion in diverse city scenes. In International Conference on Robotics and Automation, pages 4670-4677. IEEE.

Wang, J., Liu, J., and Kato, N. (2018). Networking and communications in autonomous driving: A survey. IEEE Communications Surveys \& Tutorials, 21(2):1243-1274. 